Research Article

\title{
On Analysis and Computation of Degree-Based Topological Invariants for Cyclic Mesh Network
}

\author{
Nida Zahra, ${ }^{1}$ Muhammad Ibrahim, ${ }^{1}$ Muhammad Kamran Siddiqui, ${ }^{2}$ \\ and Hajar Shooshtri $\mathbb{1}^{3}$ \\ ${ }^{1}$ Centre for Advanced Studies in Pure and Applied Mathematics, Bahauddin Zakariya University, Multan, Pakistan \\ ${ }^{2}$ Department of Mathematics, Comsats University Islamabad, Lahore Campus, Islamabad, Pakistan \\ ${ }^{3}$ Department of Mathematics, Azarbaijan Shahid Madani University, Tabriz, Iran
}

Correspondence should be addressed to Hajar Shooshtri; hajarshooshtary91@gmail.com

Received 31 May 2021; Accepted 9 July 2021; Published 15 July 2021

Academic Editor: Muhammad Kamran Jamil

Copyright (c) 2021 Nida Zahra et al. This is an open access article distributed under the Creative Commons Attribution License, which permits unrestricted use, distribution, and reproduction in any medium, provided the original work is properly cited.

Recently, there has been increasing attention on the system network due to its promising applications in parallel hanging architectures such as distributed computing (Day (2004), Day and Al-Ayyoub (2002)). Related networks differ in the circumstances of topology, and the descriptors were freshly examined by Hayat and Imran (2014) and Hayat et al. (2014). Distancebased descriptors, counting-related descriptors, and degree-based descriptors are all examples of topological descriptors. These topological characteristics are linked to chemical features of a substance, such as stability, strain energy, and boiling point. The specifications for the 1st Zagreb alpha, 1st Zagreb beta, 2nd Zagreb, sum-connectivity, geometric-arithmetic, Randic, harmonic, and atom-bond connectivity indices for mesh networks $\left(M N_{m \times n}\right)$ based on VE and EV degree are discussed in this paper.

\section{Introduction}

Cheminformatics is a relatively new field that combines chemistry, mathematics, and information science. Cheminformatics is primarily used to store, index, and retrieve information on chemicals. In index factors, graph theory is very essential. Biological activity is used as an introduction to numerous structural properties of molecules in the study of (QSAR) models. Topological indicators are a fascinating subset of these factors. Topological indices can be calculated using simply nodes (atoms) and edges in a graph representation (chemical bonds) [1,2]. A series of numbers, a polynomial, or a numeric number can all be used to identify a graph. A complete graph is represented by numbers or an array, and for those graphs, these interpretations are supposed to be unique. The topological index is a mathematical term that belongs to a graph and is unaffected by graph automorphism. It identifies the structure of the graph. Degree-based topological indices, counting-related polynomials, and graph indices are some of the most common types of topological indices in [3, 4]. A topological index is a function "Top" from $\Sigma$ to the set of real numbers. Topological indicators in a network are, of course, the number of nodes and links present in the network. Numerous networks having an atomic or molecular structure, such as honeycomb, grid networks, and hexagonal, are comparable. Topological properties of this network are very interesting, which are studied in various aspects in $[5,6]$. Hexagonal and honeycomb structures have also been identified as important in biological evolution, where intersecting triangles are critical for transmission of aid in societal problems, particularly for the development of collaboration $[7,8]$. Appropriate research on this topic and further benefit from the new research findings are given in [9-12]. While working on paraffin breaking, Wiener's approach [13] gave the impression of being a topological description. This identifier is known as the route number. After this, Wiener index is used to remember the route number. In terms of theory and practice, this topological descriptor served as the foundation for the topological index, see $[14,15]$ for details. Therefore, the topological lesions in the chemical and quantitative literature are Weiner, Zagreb, and Randic $[16,17]$. By using the previous degree concepts, all of the above works were completed. In grid history, Chellali et al. [18] developed 
two additional degree theories, namely, VE degrees and EV degrees, after some time. These concepts are a twist on the previous degree-based concept.

\section{Preliminaries}

In this section, we recall some fundamental definitions concerned to network which is usually represented by $N=(P, C)$, where $P$ is the set of points and $C$ is the set of connections of network. The degree of a point is the number of connections joined to that point. The open neighborhood, indicated as $N(v)$, for a point $v$, is written as $N(v)=u \in P \mid u, v \in C$ in networks. If we add the point $v$ to the set of $N(v)$, we get the same as the closed neighborhood of $v$, represented by $N[v]$. The number of connections that are connected to any points from the closed neighbourhood of $v$ is equal to the vertex-edge degree, denoted by $\phi_{v e}(v)$, specified in [19], of the point $v \in P$. Furthermore, the number of points in the union of the closed neighbourhoods of $u$ and $v$ is equal to the edge-vertex degree of the connection $e=u v \in C$, indicated by $\phi_{e} v(e)$ specified in [19]. Let $N$ be a simple network and $e=u v \in C(N)$. The EV and VE degree topological descriptor-related details can be seen in $[18,19]$.

Consider $N$ to be a basic network in all of the illustrations and $v \in V(N)$.

The Zagreb indices for edge-vertex degree are specified as

$$
\mathscr{M}^{e v}(N)=\sum_{e \in C(N)} \phi_{e v}(e)^{2} .
$$

The first Zagreb alpha indices for vertex-edge degree are specified as

$$
\mathscr{M}_{1}^{\alpha v e}(N)=\sum_{u \in P(N)} \phi_{v e}(u)^{2} .
$$

The first Zagreb beta indices for vertex-edge degree are specified as

$$
\mathscr{M}_{1}^{\beta v e}(N)=\sum_{u v \in C(N)}\left(\phi_{v e}(u)+\phi_{v e}(v)\right) .
$$

The second Zagreb indices for vertex-edge degree are specified as

$$
\mathscr{M}_{2}^{v e}(N)=\sum_{u v \in C(N)}\left(\phi_{v e}(u) \times \phi_{v e}(v)\right) .
$$

The Randic indices for vertex-edge degree are specified as

$$
\mathscr{R}^{v e}(N)=\sum_{u v \in C(N)}\left(\phi_{v e}(u) \times \phi_{v e}(v)\right)^{-(1 / 2)}
$$

The Randic indices for edge-vertex degree are specified as

$$
\mathscr{R}^{e v}(N)=\sum_{e \in C(N)} \phi_{v e}(e)^{-(1 / 2)} .
$$

The atom-bond connectivity indices for vertex-edge degree are specified as

$$
\mathscr{B} \mathscr{C}^{v e}(N)=\sum_{u v \in C(N)} \sqrt{\frac{\phi_{v e}(u)+\phi_{v e}(v)-2}{\phi_{v e}(u) \times \phi_{v e}(v)}} .
$$

The geometric-arithmetic ( $v e-G A)$ indices for vertexedge degree are specified as

$$
\mathscr{G} \mathscr{A}^{v e}(N)=\sum_{u v \in C(N)} \frac{2 \sqrt{\phi_{v e}(u) \times \phi_{v e}(v)}}{\phi_{v e}(u)+\phi_{v e}(v)} .
$$

The harmonic $(v e-H)$ indices for vertex-edge degree are specified as

$$
\mathscr{H}^{v e}(N)=\sum_{u v \in C(N)} \frac{2}{\phi_{v e}(u)+\phi_{v e}(v)} .
$$

The sum-connectivity (ve- $\chi$ ) indices for vertex-edge degree are specified as

$$
\chi^{v e}(N)=\sum_{u v \in C(N)}\left(\phi_{v e}(u)+\phi_{v e}(v)\right)^{-(1 / 2)}
$$

\section{Result for Mesh Network $\left(M N_{m \times n}\right)$}

In this part, we calculate several mesh network $\left(M N_{m \times n}\right)$ topological indices based on EV and VE degree, seen in Figure 1. Let $\left(M N_{m \times n}\right)$ be a mesh network. $\left(M N_{m \times n}\right)$ has $m n$ points and $2 m n-(m+n)$ connections. Table 1 shows how we partition the set of connections of $\left(M N_{m \times n}\right)$ into four components on the behalf of degrees of end points.Similarly, partition the collection of points into three components based on the degrees of points, as shown in Table 2.

Depending on the EV degree of the links of $\left(M N_{m \times n}\right)$ for $n \geq 5$, we separate them in Table 3 and partition the points and connections in Table 4 and Table 5 depending on the VE degree of $\left(M N_{m \times n}\right)$ for $n \geq 5$.

3.1. Edge-Vertex Degree-Based Indices. Now, we will calculate Zagreb and Randic indices for the mesh network $\left(M N_{m \times n}\right)$ depending on the EV degree. 


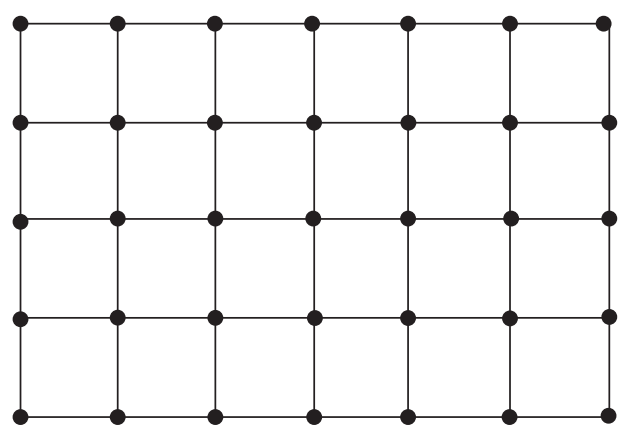

Figure 1: Mesh network $(M N)_{5 \times 7}$.

TABLE 1: Connection division of $\left(M N_{m \times n}\right)$.

\begin{tabular}{lc}
\hline No. of connections & $(\operatorname{deg}(u), \operatorname{deg}(v))$ \\
\hline 8 & $(2,3)$ \\
$2 m+2 n-12$ & $(3,3)$ \\
$2 m+2 n-8$ & $(3,4)$ \\
$2 m n-5 m-5 n+12$ & $(4,4)$ \\
\hline
\end{tabular}

TABLE 2: Points partitioning of $\left(M N_{m \times n}\right)$.

\begin{tabular}{lc}
\hline No. of points & $\operatorname{deg}(u)$ \\
\hline 4 & 2 \\
$2(m+n-4)$ & 3 \\
$m n-2(m+n)+4$ & 4 \\
\hline
\end{tabular}

TABLE 3: Link partitioning of $\left(M N_{m \times n}\right)$.

\begin{tabular}{lcc}
\hline No. of connections & Degree of end points & EV degrees \\
\hline 8 & $(2,3)$ & 5 \\
$2 m+2 n-12$ & $(3,3)$ & 6 \\
$2 m+2 n-8$ & $(3,4)$ & 7 \\
$2 m n-5(m+n)+12$ & $(4,4)$ & 8 \\
\hline
\end{tabular}

TABLE 4: Points partitioning of $\left(M N_{m \times n}\right)$.

\begin{tabular}{lcc}
\hline No. of points & Degree $(\mathrm{u})$ & VE degrees \\
\hline 4 & 2 & 6 \\
8 & 3 & 9 \\
$2(m+n)-16$ & 3 & 10 \\
4 & 4 & 14 \\
$2(m+n)-16$ & 4 & 15 \\
$m n-4(m+n)+16$ & 4 & 16 \\
\hline
\end{tabular}

TABLE 5: The VE degree of the end points of connections of $\left(M N_{m \times n}\right)$.

\begin{tabular}{lcc}
\hline No. of connections & $\begin{array}{c}\text { Degree of end } \\
\text { points }\end{array}$ & $\begin{array}{c}\text { VE degree of end } \\
\text { points }\end{array}$ \\
\hline 8 & $(2,3)$ & $(6,9)$ \\
8 & $(3,3)$ & $(9,10)$ \\
$2(m+n)-20$ & $(3,3)$ & $(10,10)$ \\
8 & $(3,4)$ & $(9,14)$ \\
$2(m+n)-16$ & $(3,4)$ & $(10,15)$ \\
8 & $(4,4)$ & $(14,15)$ \\
$2(m+n)-20$ & $(4,4)$ & $(15,15)$ \\
$2(m+n)-16$ & $(4,4)$ & $(15,16)$ \\
$2 m n-9(m+n)+40$ & $(4,4)$ & $(16,16)$ \\
\hline
\end{tabular}

3.1.1. Zagreb Index. Employing Table 3, the Zagreb index is calculated as follows:

$$
\begin{aligned}
\mathscr{M}^{e v}\left(M N_{m \times n}\right) & =\sum_{e \in E\left(M N_{m \times n}\right)} \phi_{e v}(e)^{2}, \\
\mathscr{M}^{e v}\left(M N_{m \times n}\right) & =8 \times 5^{2}+(2(m+n)-12) \times 6^{2}+(2(m+n)-8) \times 7^{2}+(2 m n-5(m+n)+12) \times 8^{2} \\
& =200+72(m+n)-432+98(m+n)-392+128 m n-320(m+n)+768 \\
& =128 m n-150(m+n)+144 .
\end{aligned}
$$

3.1.2. The Randic Index. Employing Table 3, the Randic index is calculated as follows:

$$
\begin{aligned}
\mathscr{R}^{e v}\left(M N_{m \times n}\right) & =\sum_{e \in E\left(M N_{m \times n}\right)} \phi_{e v}(e)^{-(1 / 2)} \\
\mathscr{R}^{e v}\left(M N_{m \times n}\right) & =8 \times 5^{-(1 / 2)}+(2(m+n)-12) \times 6^{-(1 / 2)}+(2(m+n)-8) \times 7^{-(1 / 2)}+(2 m n-5(m+n)+12) \times 8^{-(1 / 2)} \\
& =\frac{1}{\sqrt{2}} m n+\left(\frac{2}{\sqrt{6}}+\frac{2}{\sqrt{7}}-\frac{5}{2 \sqrt{2}}\right)(m+n)+\left(\frac{8}{\sqrt{5}}-\frac{12}{\sqrt{6}}-\frac{8}{\sqrt{7}}+\frac{6}{\sqrt{2}}\right) \\
& =0.707 m n-0.195(m+n)-0.102 .
\end{aligned}
$$


3.2. Vertex-Edge Degree-Based Indices. Now, we will calculate the 1st Zagreb alpha, 1st Zagreb beta, 2nd Zagreb, geometric-arithmetic, sum-connectivity, Randic, harmonic, and atom-bond connectivity indices for the mesh network $\left(M N_{m \times n}\right)$ depending on VE.
3.2.1. The 1st Zagreb Alpha Index. Employing Table 4, the 1st Zagreb alpha index is calculated as follows:

$$
\begin{aligned}
\mathscr{M}_{1}^{\alpha v e}\left(M N_{m \times n}\right) & =\sum_{u \in V\left(M N_{m \times n}\right)} \phi_{v e}(u)^{2} \\
\mathscr{M}_{1}^{\alpha v e}\left(M N_{m \times n}\right) & =4 \times 6^{2}+8 \times 9^{2}+(2(m+n)-16) \times 10^{2}+4 \times 14^{2}+(2(m+n)-16) \times 15^{2}+(m n-4(m+n)+16) \times 16^{2} \\
& =144+648+200(m+n)-1600+784+450(m+n)-3600+256 m n-1024(m+n)+4096 \\
& =256 m n-374(m+n)+472 .
\end{aligned}
$$

3.2.2. The 1st Zagreb Beta Index. Employing Table 5, the 1st Zagreb beta index is calculated as follows:

$$
\begin{aligned}
\mathscr{M}_{1}^{\beta v e}\left(M N_{m \times n}\right)= & \sum_{u v \in E}\left(M N_{m \times n}\right) \\
\mathscr{M}_{1}^{\beta v e}\left(M N_{m \times n}\right)= & 8 \times 15+8 \times 19+(2(m+n)-20) \times 20+8 \times 23+(2(m+n)-16) \times 25+8 \times 29 \\
& +(2(m+n)-20) \times 30+(2(m+n)-16) \times 31+(2 m n-9(m+n)+40) \times 32 \\
= & 120+152+40(m+n)-400+184+50(m+n)-400+232+60(m+n)-600 \\
& +62(m+n)-496+64 m n-288(m+n)+1280 \\
= & 64 m n-76(m+n)+72 .
\end{aligned}
$$

3.2.3. The 2nd Zagreb Index. Employing Table 5, the 2nd

Zagreb index is calculated as follows:

$$
\begin{aligned}
\mathscr{M}_{2}^{v e}\left(M N_{m \times n}\right)= & \sum_{u v \in E\left(M N_{m \times n}\right)}\left(\phi_{v e}(u) \times \phi_{v e}(v)\right), \\
\mathscr{M}_{2}^{v e}\left(M N_{m \times n}\right)= & 8 \times 54+8 \times 90+(2(m+n)-20) \times 100+8 \times 126+(2(m+n)-16) \times 150 \\
& +8 \times 210+(2(m+n)-20) \times 225+(2(m+n)-16) \times 240+(2 m n-9(m+n)+40) \times 256 \\
= & 432+720+200(m+n)-2000+1008+300(m+n)-2400+1680+450(m+n) \\
& -4500+480(m+n)-3840+512 m n-2304(m+n)+10240 \\
= & 512 m n-874(m+n)+1340 .
\end{aligned}
$$

3.2.4. The Randic Index. Employing Table 5, the Randic index is calculated as follows: 


$$
\begin{aligned}
& \mathscr{R}^{v e}\left(M N_{m \times n}\right)= \sum_{u v \in E\left(M N_{m \times n}\right)}\left(\phi_{v e}(u) \times \phi_{v e}(v)\right)^{-(1 / 2)} \\
& \mathscr{R}^{v e}\left(M N_{m \times n}\right)= 8 \times 54^{-(1 / 2)}+8 \times 90^{-(1 / 2)}+(2(m+n)-20) \times 100^{-(1 / 2)}+8 \times 126^{-(1 / 2)} \\
&+(2(m+n)-16) \times 150^{-(1 / 2)}+8 \times 210^{-(1 / 2)}+(2(m+n)-20) \times 225^{-(1 / 2)} \\
&+(2(m+n)-16) \times 240^{-(1 / 2)}+(2 m n-9(m+n)+40) \times 256^{-(1 / 2)} \\
&= \frac{1}{8} m n+\left(\frac{1}{5}+\frac{2}{5 \sqrt{6}}+\frac{2}{15}+\frac{1}{2 \sqrt{15}}-\frac{9}{16}\right)(m+n) \\
&+\left(\frac{8}{3 \sqrt{6}}+\frac{8}{3 \sqrt{10}}-2+\frac{8}{3 \sqrt{14}}-\frac{16}{5 \sqrt{6}}+\frac{8}{\sqrt{210}}-\frac{4}{3}-\frac{4}{\sqrt{15}}+\frac{5}{2}\right) \\
& 0.125 m n+0.063(m+n)+0.0241 .
\end{aligned}
$$

3.2.5. The Atom-Bond Connectivity Index. Employing Ta-

ble 5 , we calculate the above said index as follows:

$$
\begin{aligned}
& \mathscr{B} \mathscr{C}^{v e}\left(M N_{m \times n}\right)=\sum_{u v \in E\left(M N_{m \times n}\right)} \sqrt{\frac{\phi_{v e}(u)+\phi_{v e}(v)-2}{\phi_{v e}(u) \times \phi_{v e}(v)}}, \\
& \mathscr{B} \mathscr{C}^{v e}\left(M N_{m \times n}\right)=8 \times \sqrt{\frac{15-2}{54}}+8 \times \sqrt{\frac{19-2}{90}}+(2(m+n)-20) \times \sqrt{\frac{20-2}{100}}+8 \times \sqrt{\frac{23-2}{126}} \\
& +(2(m+n)-16) \times \sqrt{\frac{25-2}{150}}+8 \times \sqrt{\frac{29-2}{210}}+(2(m+n)-20) \times \sqrt{\frac{30-2}{225}} \\
& +(2(m+n)-16) \times \sqrt{\frac{31-2}{240}}+(2 m n-9(m+n)+40) \times \sqrt{\frac{32-2}{256}} \\
& =\frac{\sqrt{30}}{8} m n+\left(\frac{3 \sqrt{2}}{5}+\frac{2 \sqrt{23}}{5 \sqrt{6}}+\frac{4 \sqrt{7}}{15}+\frac{\sqrt{29}}{2 \sqrt{15}}-\frac{9 \sqrt{30}}{16}\right)(m+n) \\
& +\left(\frac{8 \sqrt{13}}{3 \sqrt{6}}+\frac{8 \sqrt{17}}{3 \sqrt{10}}-6 \sqrt{2}+\frac{8 \sqrt{3}}{3 \sqrt{2}}-\frac{16 \sqrt{23}}{5 \sqrt{6}}+\frac{24}{\sqrt{70}}-\frac{4 \sqrt{28}}{3}-\frac{4 \sqrt{29}}{\sqrt{15}}+\frac{5 \sqrt{30}}{2}\right) \\
& =0.68 m n-0.048(m+n)-0.138 .
\end{aligned}
$$

3.2.6. The Geometric-Arithmetic Index. Employing Table 5, the above said index is calculated as follows: 


$$
\begin{aligned}
\mathscr{G} \mathscr{A}^{v e}\left(M N_{m \times n}\right)= & \sum_{u v \in E\left(M N_{m \times n}\right)} \frac{2 \sqrt{\phi_{v e}(u) \times \phi_{v e}(v)}}{\phi_{v e}(u)+\phi_{v e}(v)}, \\
\mathscr{G} \mathscr{A}^{v e}\left(M N_{m \times n}\right)= & 8 \times \frac{2 \sqrt{54}}{15}+8 \times \frac{2 \sqrt{90}}{19}+(2(m+n)-20) \times \frac{2 \sqrt{100}}{20}+8 \times \frac{2 \sqrt{126}}{23} \\
& +(2(m+n)-16) \times \frac{2 \sqrt{150}}{25}+8 \times \frac{2 \sqrt{210}}{29}+(2(m+n)-20) \times \frac{2 \sqrt{225}}{30} \\
& +(2(m+n)-16) \times \frac{2 \sqrt{240}}{31}+(2 m n-9(m+n)+40) \times \frac{2 \sqrt{256}}{32} \\
= & 2 m n+\left(2+\frac{4 \sqrt{6}}{5}+2+\frac{16 \sqrt{15}}{31}-9\right)(m+n) \\
& +\left(\frac{16 \sqrt{6}}{5}+\frac{48 \sqrt{10}}{19}-20+\frac{48 \sqrt{14}}{23}-\frac{32 \sqrt{6}}{5}+\frac{16 \sqrt{210}}{29}-20-\frac{128 \sqrt{15}}{31}+40\right) \\
= & 2 m n-1.041(m+n)-0.037 .
\end{aligned}
$$

3.2.7. The Harmonic Index. Employing Table 5, the harmonic index is calculated as follows:

$$
\begin{aligned}
\mathscr{H}^{v e}\left(M N_{m \times n}\right)= & \sum_{u v \in E\left(M N_{m \times n}\right)} \frac{2}{\phi_{v e}(u)+\phi_{v e}(v)}, \\
\mathscr{H}^{v e}\left(M N_{m \times n}\right)= & 8 \times \frac{2}{15}+8 \times \frac{2}{19}+(2(m+n)-20) \times \frac{2}{20}+8 \times \frac{2}{23}+(2(m+n)-16) \times \frac{2}{25}+8 \times \frac{2}{29} \\
& +(2(m+n)-20) \times \frac{2}{30}+(2(m+n)-16) \times \frac{2}{31}+(2 m n-9(m+n)+40) \times \frac{2}{32} \\
= & \frac{1}{8} m n+\left(\frac{1}{5}+\frac{4}{25}+\frac{2}{15}+\frac{4}{31}-\frac{9}{16}\right)(m+n) \\
& +\left(\frac{16}{15}+\frac{16}{19}-2+\frac{16}{23}-\frac{32}{25}+\frac{16}{29}-\frac{4}{3}-\frac{32}{\sqrt{31}}+\frac{5}{2}\right) \\
= & 0.125 m n+0.063(m+n)+0.0241 .
\end{aligned}
$$

3.2.8. The Sum-Connectivity Index. Employing Table 5, the sum-connectivity index is calculated as follows: 


$$
\begin{aligned}
\chi^{v e}\left(M N_{m \times n}\right)= & \sum_{u v \in E\left(M N_{m \times n}\right)}\left(\phi_{v e}(u)+\phi_{v e}(v)\right)^{-(1 / 2)} \\
\chi^{v e}\left(M N_{m \times n}\right)= & 8 \times 15^{-(1 / 2)}+8 \times 19^{-(1 / 2)}+(2(m+n)-20) \times 20^{-(1 / 2)}+8 \times 23^{-(1 / 2)}+(2(m+n)-16) \times 25^{-(1 / 2)} \\
& +8 \times 29^{-(1 / 2)}+(2(m+n)-20) \times 30^{-(1 / 2)}+(2(m+n)-16) \times 31^{-(1 / 2)} \\
& +(2 m n-9(m+n)+40) \times 32^{-(1 / 2)} \\
= & \frac{1}{2 \sqrt{2}} m n+\left(\frac{1}{\sqrt{5}}+\frac{2}{5}+\frac{2}{\sqrt{30}}+\frac{2}{\sqrt{31}}-\frac{9}{4 \sqrt{2}}\right)(m+n) \\
& +\left(\frac{8}{\sqrt{15}}+\frac{8}{\sqrt{19}}-\frac{10}{\sqrt{5}}+\frac{8}{\sqrt{23}}-\frac{16}{5}+\frac{8}{\sqrt{29}}-\frac{20}{\sqrt{30}}-\frac{16}{\sqrt{31}}+\frac{10}{\sqrt{2}}\right) \\
= & 0.3533 m n-0.0194(m+n)-0.071 .
\end{aligned}
$$

\section{Conclusion}

It's crucial to explore the structure using graphs, and topological indicators are crucial for grasping the network's core topology. This sort of analysis has a wide range of applications in computer science, where different indexes based on graph invariance are used to evaluate multiple stimulation summaries. Invariants stats are essential factors for analyzing and predicating the features of chemical structures in the quantitative structure-property relationship (QSPR) and quantitative tructure-activity relationship (QSAR) explorations. We offer several finished products for VE degree and EV degree-based indices, such as the indices depending on the vertex-edge degree are the 1st Zagreb alpha, 1st Zagreb beta, 2nd Zagreb, geometric-arithmetic, sum-connectivity, Randic, harmonic, and atom-bond connectivity indices, in this article, for the mesh network $\left(M N_{m \times n}\right)$, and EV degree Randic and Zagreb indices. There will be some who are involved in designing new grids in the future, and we are examining their topological indices in order to grasp their core topology.

\section{Data Availability}

The data used to support the findings of this study are cited at relevant places within the text as references.

\section{Conflicts of Interest}

The authors declare that they have no conflicts of interest.

\section{Authors' Contributions}

All authors contributed equally to this work.

\section{References}

[1] K. Day, "Optical transpose $k$-ary $n$-cube networks," Journal of Systems Architecture, vol. 50, no. 11, pp. 697-705, 2004.
[2] K. Day and A.-E. Al-Ayyoub, "Topological properties of OTIS-networks," IEEE Transactions on Parallel and Distributed Systems, vol. 13, no. 4, pp. 359-366, 2002.

[3] P. Manuel, R. Bharati, I. Rajasingh, and C. Monica, "On minimum metric dimension of honeycomb networks," Journal of Discrete Algorithms, vol. 6, no. 1, pp. 20-27, 2008.

[4] P. Manuel and I. Rajasingh, "Minimum metric dimension of silicate networks," Ars Combinatoria, vol. 98, pp. 501-510, 2011.

[5] P. Manuel and I. Rajasingh, "Topological properties of silicate networks," in Proceedings of the 2009 5th IEEE GCC Conference \& Exhibition, vol. 15, pp. 15-25, Kuwait City, Kuwait, March 2009.

[6] B. Rajan, A. William, C. Grigorious, and S. Stephen, "On certain topological indices of silicate, honeycomb and hexagonal networks," Journal of Mathematics and Computer Science, vol. 5, pp. 530-535, 2012.

[7] S. Hayat and M. Imran, "Computation of topological indices of certain networks," Applied Mathematics and Computation, vol. 240, pp. 213-228, 2014.

[8] S. Hayat, M. Imran, and M. Y. H. Mailk, "On topological indices of certain interconnection networks," Applied Mathematics and Computation, vol. 244, pp. 936-951, 2014.

[9] M. Perc, J. Gómez-Gardeñes, A. Szolnoki, L. M. Floría, and Y. Moreno, "Evolutionary dynamics of group interactions on structured populations: a review," Journal of the Royal Society, Interface, vol. 10, 2013.

[10] M. Perc and A. Szolnoki, "Coevolutionary games-a mini review," BioSystems, vol. 99, no. 2, pp. 109-125, 2010.

[11] A. Szolnoki, M. Perc, and G. Szabó, "Topology-independent impact of noise on cooperation in spatial public goods games," Physical Review. E, Statistical, Nonlinear, and Soft Matter Physics, vol. 80, pp. 56109-56166, 2009.

[12] Z. Wang, A. Szolnoki, and M. Perc, "If players are sparse social dilemmas are too: importance of percolation for evolution of cooperation," Scientific Reports, vol. 2, no. 1, pp. 369-379, 2012.

[13] H. Wiener, "Structural determination of paraffin boiling points," Journal of the American Chemical Society, vol. 69, no. 1, pp. 17-20, 1947.

[14] A. A. Dobrynin, R. Entringer, and I. Gutman, "Wiener index of trees: theory and applications," Acta Applicandae Mathematicae, vol. 66, no. 3, pp. 211-249, 2001. 
[15] I. Gutman and O. E. Polansky, Mathematical Concepts in Organic Chemistry, Springer-Verlag, New York, NY, USA, 1986.

[16] Y. Li, F. Li, Z. Zhou, and Z. Chen, " $\mathrm{SiC}_{2}$ silagraphene and its one-dimensional derivatives: where planar tetracoordinate silicon happens," Journal of the American Chemical Society, vol. 133 , no. 4, pp. 900-908, 2010.

[17] L.-J. Zhou, Y.-F. Zhang, and L.-M. Wu, "SiC 2 siligraphene and nanotubes: novel donor materials in excitonic solar cells," Nano Letters, vol. 13, no. 11, pp. 5431-5436, 2013.

[18] M. Chellali, T. W. Haynes, S. T. Hedetniemi, and T. M. Lewis, "On ve-degrees and ev-degrees in graphs," Discrete Mathematics, vol. 340, no. 2, pp. 31-38, 2017.

[19] M. Cancan, "On edge-vertex degree and vertex-edge degree topological properties of tickysim spiking neural network," Computational Intelligence and Neuroscience, vol. 8, pp. 11-31, 2019. 